\title{
L'« exotisme » et l'« exotique » aux yeux des lexicologues et lexicographes russes
}

\section{Ekaterina Velmezova}

\section{(2) OpenEdition \\ 1 Journals}

Édition électronique

URL : http://journals.openedition.org/edl/424

DOI : $10.4000 /$ edl. 424

ISSN : 2296-5084

Éditeur

Université de Lausanne

\section{Édition imprimée}

Date de publication : 15 septembre 2009

Pagination : 173-186

ISBN : 978-2-940331-20-8

ISSN : 0014-2026

Référence électronique

Ekaterina Velmezova, "L'« exotisme » et l'« exotique » aux yeux des lexicologues et lexicographes russes », Études de lettres [En ligne], 2-3 | 2009, mis en ligne le 15 septembre 2012, consulté le 21 décembre 2020. URL : http://journals.openedition.org/edl/424 ; DOI : https://doi.org/10.4000/edl.424 


\section{L'«EXOTISME»ET L'«EXOTIQUE»AUX YEUX DES LEXICOLOGUES ET LEXICOGRAPHES RUSSES}

L'article étudie la sémantique des mots et des termes linguistiques russes èkzotizm «mot-exotisme» et èkzotičeskij "exotique». Il insiste sur l'analyse d'une composante sémantique géographique de ces lexèmes - ils renvoient à une "réalité étrangère" - et montre en même temps que, parfois, dans la lexicologie et dans la lexicographie russes, ces mots se rapportent à l'histoire. Entre autres, par les mots-exotismes sont désignés les lexèmes qui disparaissent de la langue par suite de la disparition des objets ou des phénomènes qu'ils désignent.

La linguistique se veut la discipline la plus exacte de toutes les sciences humaines, y compris dans le domaine terminologique. L'un des termes linguistiques qui s'est propagé, d'une manière intense, durant les dernières décennies en Russie et pour lequel des définitions exactes sont proposées dans les manuels et dans les dictionnaires linguistiques est èkzotizm "motexotisme " ${ }^{1}$. La notion même de "terme" suppose le caractère monosémique de ce dernier; or, si nous comparons plusieurs définitions de ce que les linguistes (ou, plus précisément, les lexicologues et les lexicographes) russes désignent par èkzotizm, nous constaterons que ce n'est pas le cas.

Dans la plupart des cas, l'èkzotizm est déterminé "géographiquement». Ainsi, dans le célèbre dictionnaire de terminologie linguistique de D. Rozental et M. Telenkova, le lexique exotique (èkzotičeskaja leksika) est défini comme "les mots et les expressions empruntés à d'autres langues (souvent peu connues) et utilisés pour donner une couleur

I. Précisons tout de suite que cette notion ne fait pas partie des termes linguistiques principaux, c'est pourquoi elle n'est pas mentionnée dans certains livres linguistiques. 
locale au discours (reč) $)^{2}$. Dans le Dictionnaire encyclopédique linguistique (Lingvističeskij ènciklopedičeskij slovar'), le mot èkzotizm se révèle synonyme des termes ètnografizm et regionalizm. Ces derniers sont mentionnés sous l'entrée "Emprunt" (Zaimstvovanie), rédigée par I. Dobrodomov. L’emprunt y est déterminé comme un «élément d'une langue étrangère", par exemple un mot, un morphème, une construction syntaxique transposés d'une langue à une autre à la suite de contacts linguistiques. Sont distingués ensuite, d'un côté, les emprunts complètement assimilés par telle ou telle langue, de l'autre, les emprunts qui gardent les traces phonétiques, grammaticales et sémantiques de leurs origines étrangères. C'est dans ce dernier groupe que nous trouvons ce qui est désigné comme èkzotizm: ces mots renvoient à des notions qui sont propres à des peuples ou à des pays étrangers. En guise d'exemple d'èkzotizm, dans le Dictionnaire encyclopédique linguistique, nous trouvons le mot guajava, "goyavier", qui désigne un arbre fruitier d'Amérique tropicale. Comme il est indiqué par la suite, les interprétations de ces mots sont le plus souvent proposées dans les dictionnaires de mots étrangers, et on les trouve beaucoup plus rarement dans les dictionnaires de caractère plus général ${ }^{3}$.

L'exemple susmentionné d'une plante exotique est révélateur d'une tendance particulière. En passant de l'analyse sémantique du «terme» linguistique èkzotizm à l'étude de l'interprétation de l'èkzotizm en tant que mot de la langue russe ${ }^{4}$ (avec, en même temps, l'étude de la sémantique des mots èkzotika "exotisme», èkzotičeskij "exotique" ${ }^{5}$,

2. D. Rozental' et M. Telenkova, Slovar'-spravočnik lingvističeskih terminov, p. 391392.

3. I. Dobrodomov, «Zaimstvovanie», p. 158.

4. A quelques exceptions près, nous ne trouvons pas le mot èkzotizm dans les dictionnaires raisonnés de la langue russe - et cela à la différence des dictionnaires d'autres types, comme, par exemple, le Dictionnaire russe de formation des mots (A. Tihonov, Slovoobrazovatel'nyj slovar' russkogo jazyka, vol. 2, p. 417), etc. A titre d'exception, référons-nous au Grand Dictionnaire des mots étrangers (A. Bulyko, Bol'soj slovar' inojazyčnyh slov, p. 673), où l'èkzotizm est défini comme: 1) «la même chose qu'èkzotika "exotisme»»; 2) (avec la note «lingv.» [lingvističeskoe «linguistique»]) "un mot emprunté qui désigne un objet ou une notion propre à la vie d'autres peuples». Dans le Dictionnaire de la langue littéraire russe moderne, la deuxième signification de ce mot n'est pas indiquée: l'èkzotizm y serait la même chose que èkzotika (Slovar'sovremennogo russkogo literaturnogo jazyka, vol. 17, p. 1744).

5. En sortant des limites de la lexicographie linguistique proprement dite, mentionnons ici un certain nombre d'entrées des encyclopédies et des dictionnaires 
etc.), soulignons que, dans la plupart des dictionnaires raisonnés ${ }^{6}$, en guise d'exemples sous les entrées èkzotika ou èkzotičeskij, ce sont précisément les plantes qui sont citées. En quelque sorte, cela pourrait "se justifier" par l'histoire de ce mot. Le mot même d'èkzotizm (qui est d'origine grecque et qui signifie "étranger») est entré dans la langue russe assez tard, dans la deuxième moitié du XIX siècle $^{7}$, à partir de l'allemand ${ }^{8}$. En ce qui concerne les dictionnaires russes, ce mot se trouve pour la première fois dans le Dictionnaire complet des mots étrangers qui sont entrés dans la langue russe (composé d'après le modèle du dictionnaire allemand de Heyse) (Polnyj slovar' inostrannyh slov, vošedših v sostav russkogo jazyka [sostavlennyj po obrazcu nemeckogo slovarja Gejze]) (Saint-Pétersbourg, 1861) 9. Dans le Dictionnaire raisonné de la langue grand-russe vivante de V. Dahl (dont la première édition date de 1863 1866), le mot èkzotičeskij est défini comme "étranger (čužezemnyj, inozemnyj), des pays chauds, cf. la végétation [exotique]» ${ }^{10}$. Par la suite ce sont les plantes qui sont devenues l'un des exemples préférés des auteurs des dictionnaires russes à la recherche d'illustrations linguistiques de ce qui, en principe, pourrait être exotique: référons-nous au moins aux

encyclopédiques qui contiennent le mot èkzotičeskij "exotique»: roches exotiques (èkzotičeskie skaly) (A. Granat et I. Granat éd., Enciklopedičeskij slovar russkogo bibliografičeskogo instituta brat'ev A.N. i I.N. Granat, vol. 51, p. 184), chat exotique à poil ras (èkzotičeskaja korotkošerstnaja koška), atome exotique (èkzotičeskij atom), rocher exotique (èkzotičeskij utes) (S. Kondrat'ev éd., Bol'šaja ènciklopedija, vol. 60, p. 108-109)... Dans cet article, nous ne nous arrêterons pas en détail sur la présentation des mots èkzotika, ékzotičeskij, èkzot et èkzotizm dans les dictionnaires des différents types, nous nous limiterons au domaine de la linguistique.

6. La quantité très importante de dictionnaires du russe de tous les types et d'encyclopédies de toutes sortes qui a été éditée en Russie durant les dernières décennies rend pratiquement impossible une description complète et exhaustive de la représentation des mots correspondants par les lexicographes russes; cela aurait été possible il y a quelques années (cf., par exemple, R. Rogožnikova, Svodnyj slovar' sovremennoj russkoj leksiki, vol. 2, p. 712).

7. Il existe un autre point de vue qui «retarde» l'apparition de ce mot en russe d'au moins plusieurs décennies: d'après A. Guskova et B. Sotine, le mot èkzotika n'a été emprunté en russe "des langues de l'Europe de l'Ouest" qu'au début du XXe siècle (A. Gus'kova et B. Sotin, Populjarnyj slovar' russkogo jazyka [tolkovo-ènciklopedičeskij], p. 836).

8. L. Krysin, Tolkovyj slovar' inojazyčnyh slov, p. 904.

9. P. Černyh, Istoriko-ètimologičeskij slovar' sovremennogo russkogo jazyka, vol. II, p. 440 .

Io. V. Dal', Tolkovyj slovar' živogo velikorusskogo jazyka, vol. 4, p. 1529. 
"plantes exotiques" (èkzotičeskie rastenija), qui sont mentionnées dans de nombreux dictionnaires ${ }^{11}$, ainsi qu'à la "végétation exotique» ${ }^{12}$, etc.

D'autre part, dès le dictionnaire de Dahl, la composante "climatique" du mot èkzotičeskij a été distinguée de façon très claire: il s'agissait impérativement d'un climat chaud, ce qui sous-entendait que le nord ne pouvait pas être considéré comme exotique. Dans les dictionnaires plus récents, la «composante climatique» dans la sémantique de cet adjectif russe devient moins évidente, sans pourtant disparaître complètement. Par exemple, dans le Dictionnaire raisonné de la langue russe de $\mathrm{D}$. Ušakov, en guise d'exemple de l'interprétation du syntagme èkzotičeskie strany, "pays exotiques", sont indiqués les "pays tropicaux du point de vue d'un Européen " ${ }^{13}$, tandis que dans le Grand Dictionnaire des mots étrangers, en guise d'exemple d' "exotisme» est citée "la nature insolite [...] des pays du Sud du point de vue des habitants du Nord ${ }^{14}$. Dans le Dictionnaire des mots étrangers de I. Lexin et F. Petrov, le mot èkzotika "exotisme» est défini comme «certains objets, phénomènes ou traits particuliers qui sont typiques de régions et de pays lointains, par exemple de pays et de régions de l'Est et du Sud [...]» ${ }^{15}$. De nombreux dictionnaires de la langue russe proposent, à titre d'exemple de syntagme «typique» pour le mot èkzotika, précisément «l'exotisme oriental» (vostočnaja èkzotika) ${ }^{16}$. Ainsi, il n'y aurait pas plus d'«exotisme» ${ }^{17}$ à l'ouest qu'au nord (cf. plus haut), même si, évidemment, la

II. A. Bulyko, Bol'šoj slovar' inojazyčnyh slov, p. 674; A. Evgen'eva, Slovar' russkogo jazyka, vol. 4, p. 748 ; V. Lopatin et L. Lopatina, Malyj tolkovyj slovar' russkogo jazyka, p. 683; V. Lopatin et L. Lopatina, Russkij tolkovyj slovar', p. 808; S. Ožegov, Slovar' russkogo jazyka, p. 739; Slovar' sovremennogo russkogo literaturnogo jazyka, vol. 17, p. 1746.

I2. D. Ušakov, Tolkovyj slovar' russkogo jazyka, vol. 4, p. 1398.

13. Ibid.

I4. A. Bulyko, Bol'šoj slovar' inojazyčnyh slov, p. 673-674.

I5. I. Lexin et F. Petrov, Slovar' inostrannyh slov, p. 586, cf. aussi S. Kondrat'ev, Bol'śaja ènciklopedija, vol. 60, p. 108.

I6. A. Gus'kova et B. Sotin, Populjarnyj slovar' russkogo jazyka (tolkovoènciklopedičeskij), p. 836; L. Krysin, Tolkovyj slovar' inojazyčnyh slov, p. 294; V. Lopatin et L. Lopatina, Russkij tolkovyj slovar', p. 808; V. Makarenko et al., Kratkij slovar' sovremennyh ponjatij i terminov, p. 487; S. Ožegov, Slovar' russkogo jazyka, p. 739; A. Semenjuk et al., Leksičeskie trudnosti russkogo jazyka. Slovar'-spravočnik, p. 562.

17. C'est pourquoi cela ne doit pas être un hasard si dans certains dictionnaires, en guise d'exemples «littéraires» (tirés de la littérature russe) de syntagmes qui contiennent 
réalité géographique ne coïncide pas toujours avec ses représentations chez les locuteurs de différentes langues. D’autre part, cela signifie que les plantes exotiques (èkzotičeskie rastenija), si aimées des lexicographes russes, ne poussent que dans les régions (exotiques) du Sud ou de l'Est.

Le mot de même racine èkzot(y) (qui est entré en russe en suivant le même chemin qu'èkzotika ${ }^{18}$ ) renvoie non seulement à des plantes, mais aussi à des «animaux importés de pays dont le climat est nettement différent par rapport au climat des pays où ils ont été importés " ${ }^{19}$. Ainsi, la représentation "lexicographique» de l'«exotique» en russe "s'élargit»: un autre exemple fréquent de ce qui pourrait être "exotique» est, pour les auteurs des dictionnaires russes, la nature en général, ce qui inclut non seulement la flore, mais aussi la faune ${ }^{20}$ - référons-nous sous

le mot èkzotičnyj "exotique», nous trouvons précisément la description du climat «du Sud-Est", "chaud et humide» (A. Evgen'eva, Slovar' russkogo jazyka, vol. 4, p. 748; Slovar' sovremennogo russkogo literaturnogo jazyka, vol. 17, p. 1746). En même temps, c'est l'Inde, un pays "du Sud-Est», qui est cité en exemple comme pays «exotique» dans le Dictionnaire de la langue littéraire russe moderne (Slovar' sovremennogo russkogo literaturnogo jazyka, vol. 17, p. 1746).

I8. L. Krysin, Tolkovyj slovar' inojazyčnyh slov, p. 904.

19. I. Lehin, F. N. Petrov, Slovar' inostrannyh slov, p. 586; cf. aussi A. Bulyko, Bol’soj slovar' inojazyčnyh slov, p. 674; L. Krysin, Tolkovyj slovar' inojazyčnyh slov, p. 904, etc.

20. A. Bulyko, Bol'šoj slovar' inojazyčnyh slov, p. 673; B. Vvedenskij (éd.), Malaja sovetskaja ènciklopedija, vol. 10, p. 738; A. Gorkin (éd.), Illjustrirovannyj ènciklopedičeskij slovar', p. 793; A. Gus'kova et B. Sotin, Populjarnyj slovar' russkogo jazyka (tolkovo-ènciklopedičeskij), p. 836; A. Evgen'eva, Slovar' russkogo jazyka, vol. 4, p. 748; T. Efremova, Novyj slovar' russkogo jazyka (tolkovo-slovoobrazovatel'nyj), vol. 2, p. 1045 ; V. Makarenko et al., Kratkij slovar' sovremennyh ponjatij i terminov, p. 487; A. Prohorov (éd.), Sovetskij ènciklopedičeskij slovar', p. 1542; A. Prohorov, Bol'šoj rossijskij ènciklopedičeskij slovar', p. 1824; A. Semenjuk et al., Leksičeskie trudnosti russkogo jazyka. Slovar'-spravočnik, p. 562; Slovar' sovremennogo russkogo literaturnogo jazyka, vol. 17, p. 1744; D. Ušakov, Tolkovyj slovar' russkogo jazyka, vol. 4, p. 1398. Ainsi, c'est un animal particulier (le chameau) qui vient en premier dans l'exemple littéraire que les auteurs de plusieurs dictionnaires russes citent le plus souvent pour le mot èkzotika - une citation du livre Le Veau d'or (Zolotoj telenok) de I. Ilf et E. Petrov: «[...] après Orenbourg [...] ils aperçurent le premier chameau, la première yourte et le premier Kazakh en haut bonnet pointu [...] C'était le début de l'exotisme: les vaisseaux du désert, les libres fils des steppes et autre bétail de trait» (" [Za Orenburgom] oni uvideli pervogo verbljuda, pervuju jurtu i pervogo kazaha v ostrokonečnoj mehovoj šapke... Načalas' èkzotika, korabli pustyni, vol'noljubivye syny stepej i pročee romantičeskoe tjaglo») (cité d'après Slovar' sovremennogo russkogo literaturnogo jazyka, vol. 17, p. 1744 [traduit en français par A. Préchac: I. Ilf et E. Pétrov, Le Veau d’or, p. 378-379]; cf. aussi 
ce rapport à la définition suivante de l'èkzot: «Spécimen de plante ou d'animal exotique " ${ }^{21}$. Si dans certaines définitions de l'èkzot, la composante "climatique» de la sémantique de ce mot n'est que sous-entendue (ce sont «les plantes et les animaux importés qui ne sont pas typiques de la flore et de la faune locales » ${ }^{22}$ ), dans d'autres définitions elle est spécifiée de façon plus détaillée: il s'agit d'une plante ou d'un animal «qui n'est pas typique de la localité en question, qui est importé d'ailleurs (généralement d'une région subtropicale ou tropicale)» ${ }^{23}$. Cela voudrait dire que, comme dans le cas de l'èkzotika, les plantes et les animaux septentrionaux ne peuvent pas être considérés comme des èkzoty. Pour confirmer cette hypothèse référons-nous à une définition plus détaillée de l'èkzot qui contient plusieurs exemples (il s'agit de plantes importées du Sud ou de l'Est) et qui est proposée dans la Grande Encyclopédie (Bol'šaja ènciklopedija) publiée en 2006: sont considérées comme èkzoty des espèces importées de plantes et d'animaux qui ne sont pas typiques de la flore et de la faune locales. D'ordinaire [souligné par nous - E.V.] cela sous-entend des plantes tropicales et subtropicales importées dans des pays à climat tempéré, mais aussi des plantes des pays froids importées dans des régions tropicales [...]. Certains èkzoty s'acclimatent bien dans leurs nouvelles conditions et peuvent se propager ensuite sans l'aide de l'homme. Ainsi, les mimosas (d'Afrique), les eucalyptus (d'Australie), les arbrisseaux à thé (de Chine), les yuccas et les agaves (du Mexique) qui ont été importés sur le littoral de la mer Noire, se sont bien adaptés aux conditions locales et se reproduisent [actuellement] par semence et végétativement ${ }^{24}$.

Et voici un exemple plus rare - pas aussi typique du point de vue sémantique (la composante "climatique» de la signification de ce mot y est plutôt cachée) - de l'utilisation du mot èkzot dans la littérature:

A. Gus'kova et B. Sotin, Populjarnyj slovar' russkogo jazyka [tolkovo-ènciklopedičeskij], p. 836; A. Semenjuk et al., Leksičeskie trudnosti russkogo jazyka. Slovar'-spravočnik, p. 562). Cf. aussi les exemples littéraires contenant des "rossignols» (solov'i) et des «roses» (rozy) en qualité d'exotismes (A. Evgen'eva, Slovar' russkogo jazyka, vol. 4, p. 748), ainsi que le tigre, un "animal exotique» (ibid.; Slovar' sovremennogo russkogo literaturnogo jazyka, vol. 17, p. 1746).

2I. D. Ušakov, Tolkovyj slovar' russkogo jazyka, vol. 4, p. 1398.

22. B. Vvedenskij, Malaja sovetskaja ènciklopedija, vol. 10, p. 738.

23. T. Efremova, Novyj slovar' russkogo jazyka (tolkovo-slovoobrazovatel'nyj), vol. 2, p. 1045, cf. aussi Slovar' sovremennogo russkogo literaturnogo jazyka, vol. 17, p. 1744.

24. S. Kondrat'ev, Bol'śaja ènciklopedija, vol. 60, p. 109. 
Piotr Maximovitch était anxieux pour [...] les pépinières d'exôtikos, arbres d'outre-mer: pin de Douglas, peuplier du Canada et pin de Murray ${ }^{25}$.

Pour revenir à la terminologie linguistique, soulignons que les «motsexotismes" (slova-èkzotizmy) comme type particulier d'emprunts (avec une composante sémantique "géographique" par excellence) possèdent une série de traits qui les distinguent des autres mots de la langue russe (de nombreuses recherches linguistiques ont déjà été consacrées à ce sujet). Ainsi, d'après E. Marinova, il faut distinguer les «motsexotismes" (èkzotizmy) et les néologismes (neologizmy) conformément à la présence ou à l'absence d'une dénotation correspondante dans la réalité décrite par la langue. La présence d'une dénotation désigne un néologisme, tandis que son absence caractérise un "mot-exotisme». En même temps, la frontière entre les "mots-exotismes» et les néologismes n'est pas stable, et les exemples sont nombreux dans le langage de la réalité post-soviétique:

[...] avant la perestroïka, on considérait comme «mots-exotismes» les lexèmes comme bistro [cf. bistro - E.V.], gastarbajter [cf. der Gastarbeiter "travailleur immigré" - E.V.], dollar, impressario [cf. impresario «imprésario" - E.V.], kolledž [cf. college "établissement d'enseignement supérieur»; cf. aussi collège - E.V.], krup'e [cf. croupier - E.V.], lenč (lanč) [cf. lunch «déjeuner»-E.V.], marka [cf. der Mark «mark» - E.V.], mafija [cf. Mafia "maffia» - E.V.], piccerija [cf. pizzeria - E.V.], spiker [cf. speaker "conférencier, intervenant"E.V.], uikend [cf. weekend "week-end"-E.V.], šop [cf. shop «magasin» - E.V.], et on les utilisait tels quels, sous ces formes (avant tout dans la littérature qu'on traduisait en russe). Maintenant ces mots se sont "russifiés», et certains d'entre eux ont déjà acquis une nouvelle signification sur le sol russe $[\ldots]^{26}$.

25. «Petr Maksimovič volnovalsja iz-za posadok [...] “èkzotov” - skororastuščih zamorskih derev'ev: duglasovoj pihty, kanadskogo topolja i [...] sosny Murreja» (K. Paustovskij, Povest'o lesah, cité d'après: Slovar' sovremennogo russkogo literaturnogo jazyka, vol. 17, p. 1744; traduit en français par L. Delt et M. Deniaud [C. Paoustovski, Le Roman des forêts, p. 220]).

26. E. Marinova, «Èkzotičeskaja leksika kak lingvističeskij fenomen», p. 138-139. 
Dans leur grande majorité, les "mots-exotismes" sont des substantifs concrets qui désignent souvent des êtres animés. Leur particularité stylistique consiste dans le fait qu'ils ne sont pratiquement pas utilisés dans le langage parlé. Du point de vue de la dérivation des mots, les "mots-exotismes» ne sont pas productifs (hormis leur capacité à servir, souvent, à la formation d'adjectifs dits relatifs). De plus, l'augmentation de la capacité à former des mots à partir d'un "mot-exotisme» indique que le lexème correspondant ne devrait plus être considéré comme «exotique", qu'il ne désigne plus une notion d'une autre culture et qu'on l'utilise déjà au sens figuré dans la langue qui l'avait emprunté (comme, par exemple, le mot zombi «zombie», duquel en russe sont dérivés les mots zombirovat", "transformer en zombie", zombirovanie, "(processus de) la transformation en zombie», zombirovannyj, «transformé en zombie», etc.). Un trait typique de la sémantique des «mots-exotismes» est leur caractère monosémique:

Sont, seuls, polysémiques les «mots[-exotismes]» qui l'étaient déjà dans la langue-source. Par exemple, le mot arroba «arrobe» a deux sens: "unité de mesure de masse dans certains pays d'Amérique Latine» et "unité de mesure de volume et de capacité des matières sèches et liquides dans les mêmes pays " ${ }^{27}$.

Les liens syntagmatiques et paradigmatiques des exotismes dans la langue sont très faibles. Enfin graphiquement, les exotismes en russe sont souvent mis en italique ou écrits en caractères latins. Au titre de trait universel, on pourrait indiquer des variations fréquentes dans l'orthographe des exotismes, ce qui est lié à des variations dans leur phonétique (dans leur prononciation) ${ }^{28}$.

Enfin, la notion de «mot-exotisme» est liée à celles de lacune sémantique et de lacune dénotative, introduites par les ethno-psycholinguistes russes (il suffit de mentionner les recherches de J. Sorokine et de ses élèves ${ }^{29}$ ):

27. Ibid., p. 140, cf. aussi S. Timina, «Èkzotičeskaja leksika v diskurse sovremenoj rossijskoj pressy", p. 196.

28. S. Timina, «Fonetiko-grafičeskie osobennosti èkzotizmov (na materiale jazyka sovremennoj anglojazyčnoj pressy)».

29. G. Bykova, «Lakuny v jazykovoj kartine mira amurčan»; N. Glazačeva, «Lakuny i teorija mežkul'turnoj kommunikacii»; O. Pylaeva, "Otnositel'nye ètnografičeskie lakuny"; O. Titova, "Bezèkvivalentnaja leksika anglijskogo jazyka v lingvokul'turnom aspekte (na primere zaimstvovanija v'etnamskih realij) », etc. 
Les lacunes sont [...] les synonymes des objets, des phénomènes, des processus et des états spécifiques qui contredisent (protivorečit') l'expérience usuelle (uzual'nyj opyt) des représentants d'une autre langue et d'une autre culture. Parmi les traits principaux de la lacune, mentionnons son caractère non compréhensible (neponjatnost'), non habituel (neprivyčnost') (exotique [èkzotičnost'] [souligné par nous - E.V.]), l'étrangeté (čuždost', neznakomost') des objets correspondants pour la personne en question (recipient).

Et cela vaut, en général, pour la «base cognitive commune», qui est la même pour les «représentants d'une seule et même communauté culturelle» et qui contient les «invariants de la perception des objets culturels déterminés par/ pour chaque nation (nacional’no determinirovannye)» ${ }^{30}$.

Ces lacunes existent dans la langue qui emprunte un exotisme et elles «témoignent du caractère abondant ou non suffisant de l'expérience d'une certaine communauté linguistique et culturelle par rapport à une autre communauté» ${ }^{31}$. A l'égard des exotismes, il peut parfois s'agir de lacunes relatives:

L'exotisme est une expression linguistique manifeste de la faible propagation d'un objet dans la vie quotidienne - il s'agit d'un mot qui n'est pas sémantiquement assimilé, d'une [...] lacune relative ${ }^{32}$.

Ainsi tout semble clair: en russe, l'«exotisme» et l'« exotique» - mots et termes - sont définis, avant tout, "géographiquement", renvoyant à ce qui est propre à des peuples, à des cultures ou à des pays étrangers.

Or il existe d'autres définitions des "(mots-)exotismes" qui sont plus inattendues. Par exemple, dans le célèbre manuel universitaire de linguistique de J. Maslov Introduction à la linguistique (Vvedenie v jazykoznanie), le "mot-exotisme», comme désignation d'une réalité étrangère, a des connotations historiques par excellence. Le terme "mot-exotisme» (èkzotizm) y est introduit dans le chapitre sur les changements historiques du vocabulaire ${ }^{33}$ et ne se rapporte plus aux mots-emprunts, mais aux mots-«historismes» (slova-istorizmy). C'est ainsi que sont désignés

30. N. Glazačeva, "Lakuny i teorija mežkul'turnoj kommunikacii», p. 31-33.

3I. J. Sorokin et I. Markovina, «Kul'tura i ee psiholingvističeskaja cennost'», p. 8.

32. O. Pylaeva, "Otnositel'nye ètnografičeskie lakuny», p. 57.

33. J. Maslov, Vvedenie v jazykoznanie, p. 212-229. 
les mots qui disparaissent de la langue par suite de la disparition des objets ou des phénomènes qu'ils désignent ${ }^{34}$ :

Les «mots-historismes» (istorizmy) sont toujours utilisés quand il s'agit du passé, ainsi que dans un contexte spécifique "muséal» (muzejnyj kontekst). Certains [...] mots [russes] [soha «araire», berdy̌s «francisque", kolčan "carquois", diližans "diligence», konka «omnibus", prikaz "département", graf "comte», statskij sovetnik "conseiller d'Etat", predvoditel' dvorjanstva "maréchal de la noblesse», gorodovoj "sergent de ville», barin "seigneur», lakej "laquais»"35] sont devenus, dans leurs sens propres, mots-«historismes» (ou «mots-exotismes» [èkzotizmy] - désignations d'une réalité étrangère), mais ils sont toujours utilisés dans la langue dans leur sens figuré, souvent avec des connotations négatives (par exemple, les mots barin, lakej) ${ }^{36}$.

Ainsi, même si le "mot-exotisme» renvoie toujours à une "désignation d'une réalité étrangère", il peut s'agir non (seulement) de la géographie, mais (également) de l'histoire. Un "mélange» encore plus évident de l'histoire et de la géographie apparaît dans la sémantique de la notion de "langues exotiques" (èkzotičeskie jazyki), très fréquente dans les travaux se rapportant au domaine de l'histoire des idées linguistiques en Russie et en Union soviétique. Ce sujet mériterait une étude particulière.

Ekaterina Velmezova

Lausanne

34. Les mots-«historismes» (istorizmy) sont opposés ici aux mots-«archaïsmes» (arhaizmy): ces derniers renvoient à des mots qui ont disparu de la langue malgré le fait que les objets ou les phénomènes qu'ils désignent existent toujours.

35. Cf. J. Maslov, Vvedenie v jazykoznanie, p. 213. - E.V.

36. Ibid., p. 214. 


\section{BIBLIOGRAPHIE}

Bulyко, A., Bol'šoj slovar' inojazyčnyh slov, Moskva, Martin, 2004.

Byкоva, G., "Lakuny v jazykovoj kartine mira amurčan", in Lakuny v jazyke i reči. Sbornik naučnyh trudov, éd. J. Sorokin, Blagoveščensk, Izdatel'stvo BGPU, 2005, fasc. 2, p. 25-31.

ČERnYH, P., Istoriko-ètimologičeskij slovar' sovremennogo russkogo jazyka, vol. I-III, Moskva, Russkij jazyk, 1993.

DAL' [DAL], V., Tolkovyj slovar' živogo velikorusskogo jazyka (reprint de l'édition de 1903-1904, sous la dir. de Ivan Boduèn de Kurtenè [Baudoin de Courtenay]), Moskva, Progress-Univers, 1994.

Doвrodomov, I., "Zaimstvovanie», in Lingvističeskij ènciklopedičeskij slovar', éd. V. Jarceva, Moskva, Sov. ènciklopedija, 1990, p. $158-159$.

Efremova, T., Novyj slovar' russkogo jazyka (tolkovo-slovoobrazovatel'nyj), Moskva, Russkij jazyk, 2000, vol. 1-2.

Evgen'eva, A., Slovar' russkogo jazyka, Moskva, Russkij jazyk, Poligrafresursy, 1981-1984, vol. 1-4.

GlazAČEVA, N., «Lakuny i teorija mežkul'turnoj kommunikacii», in Lakuny v jazyke i reči, Sbornik naučnyh trudov, éd. J. Sorokin, Blagoveščensk, Izdatel'stvo BGPU, 2005, fasc. 2, p. 31-34.

Gorkin, A. (éd.), Illjustrirovannyj ènciklopedičeskij slovar', Moskva, Bol'šaja Rossijskaja ènciklopedija - Izdatel'skij dom Èkonomgazeta, 1995 [Dictionnaire encyclopédique illustré].

Granat, A. et Granat, I. (éd.), Ėnciklopedičeskij slovar russkogo bibliografičeskogo instituta brat'ev A.N. i I.N. Granat, Moskva, Red. Russkgo bibliografičeskogo Instituta Granat, 1910-1948.

Gus'rova, A. et Sotin, B., Populjarnyj slovar' russkogo jazyka (tolkovoènciklopedičeskij), Moskva, Russkij jazyk. Media, 2003.

Ilf, I. et Pétrov, E., Le Veau d'or (trad. A. Préchac), Paris, Scarabée et Compagnie, 1984. 
Kondrat'ev, S. (éd.), Bol’šaja ènciklopedija, 62 vols, Moskva, Terra, 2006.

Krysin, L., Tolkovyj slovar' inojazyčnyh slov, Moskva, Èksmo, 2007.

Lehin, I. et Petrov, F., Slovar' inostrannyh slov, Moskva, Russkij jazyk, 1981.

Lopatin, V. et Lopatina, L., Malyj tolkovyj slovar' russkogo jazyka, Moskva, Russkij jazyk, 1990.

—, Russkij tolkovyj slovar', Moskva, Russkij jazyk, 1994.

Makarenko, V. et al., Kratkij slovar' sovremennyh ponjatij i terminov, Moskva, Respublika, 1995.

Marinova, E., "Ėkzotičeskaja leksika kak lingvističeskij fenomen», Vestnik Nižegorodskogo universiteta im. N.I. Lobačevskogo, Serija filologija, 1 (2003), p. 138-142.

Maslov, J., Vvedenie v jazykoznanie, Sankt-Peterburg, Filologičeskij fakul'tet SPbGU - Moskva, Akademija, 2005.

Ožegov, S., Slovar' russkogo jazyka, Moskva, Russkij jazyk, 1988.

Paoustovski, C., Le Roman des forêts, suivi de Le Pays de la Mestchiora (trad. L. Delt et M. Deniaud), [Paris], Gallimard, 1971.

Prohorov, A. (éd.), Sovetskij ènciklopedičeskij slovar', Moskva, Sovetskaja ènciklopedija, 1986.

—, Bol'šoj rossijskij ènciklopedičeskij slovar', Moskva, Bol'šaja Rossijskaja ènciklopedija, 2006.

Pylaeva, O., "Otnositel'nye ètnografičeskie lakuny», in Lakuny v jazyke i reči. Sbornik naučnyh trudov, éd. J. Sorokin, Blagoveščensk, Izdatel'stvo BGPU, 2005, fasc. 2, p. 55-60.

Rogožnikova, R., Svodnyj slovar' sovremennoj russkoj leksiki, Moskva, Russkij jazyk, 1991, vol. 1-2.

Rozental', D. et Telenkova, M., Slovar'-spravočnik lingvističeskih terminov, Moskva, Prosveščenie, 1985.

Semenjuk, A. et al., Leksičeskie trudnosti russkogo jazyka. Slovar'spravočnik, Moskva, Russkij jazyk, 1994.

Slovar' sovremennogo russkogo literaturnogo jazyka, Moskva/Leningrad, Izd. Akademii nauk SSSR, 1948-1965, vol. 1-17.

Sorokin, J. et Markovina, I., "Kul'tura i ee psiholingvističeskaja cennost' ", in Etnopsiholingvistika, éd. J. Sorokin, Moskva, Nauka, 1988.

Tinonov, A., Slovoobrazovatel'nyj slovar' russkogo jazyka, Moskva, Russkij jazyk, 1985, vol. 1-2. 
TiminA, S., "Èkzotičeskaja leksika v diskurse sovremennoj rossijskoj pressy", in Russkaja filologija i metodika prepodavanija russkogo jazyka, Tajbèj, Izdatel'stvo Tamkanskogo universiteta, 2005, p. 193-201.

—, «Fonetiko-grafičeskie osobennosti èkzotizmov (na materiale jazyka sovremennoj anglojazyčnoj pressy)», http://www.lingvomaster. ru/files/197.pdf

Titova, O., «Bezèkvivalentnaja leksika anglijskogo jazyka v lingvokul'turnom aspekte (na primere zaimstvovanija v'etnamskih realij", in Lakuny v jazyke i reči. Sbornik naučnyh trudov, éd. J. Sorokin, Blagoveščensk, Izdatel'stvo BGPU, 2005, fasc. 2, p. 76-85.

UšAkov, D., Tolkovyj slovar' russkogo jazyka, Moskva, Astrel' - AST, 2000, vol. 1-4.

Vvedenskij, B. (éd.), Malaja sovetskaja ènciklopedija, Moscou, Sovetskaja ènciklopedija, 1958-1961, vol. 1-10. 
